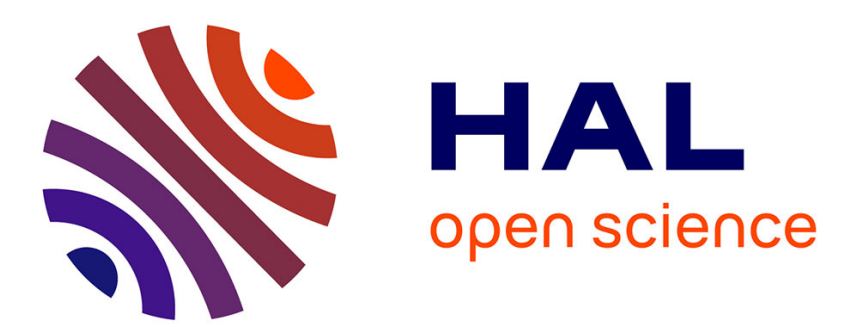

\title{
Optimization of the Growth Conditions for High Quality CH 3 NH 3 PbBr 3 Hybrid Perovskite Single Crystals
}

\author{
Smail Amari, Jean-Marie Verilhac, Eric Gros D'aillon, Alain Ibanez, Julien
} Zaccaro

\section{- To cite this version:}

Smaïl Amari, Jean-Marie Verilhac, Eric Gros D'aillon, Alain Ibanez, Julien Zaccaro. Optimization of the Growth Conditions for High Quality $\mathrm{CH} 3 \mathrm{NH} 3 \mathrm{PbBr} 3$ Hybrid Perovskite Single Crystals. Crystal Growth \& Design, 2020, 20 (3), pp.1665-1672. 10.1021/acs.cgd.9b01429 . hal-02459019

\section{HAL Id: hal-02459019 https://hal.science/hal-02459019}

Submitted on 22 Sep 2020

HAL is a multi-disciplinary open access archive for the deposit and dissemination of scientific research documents, whether they are published or not. The documents may come from teaching and research institutions in France or abroad, or from public or private research centers.
L'archive ouverte pluridisciplinaire HAL, est destinée au dépôt et à la diffusion de documents scientifiques de niveau recherche, publiés ou non, émanant des établissements d'enseignement et de recherche français ou étrangers, des laboratoires publics ou privés. 


\title{
Optimization of the growth conditions for high
}

\section{quality $\mathrm{CH}_{3} \mathrm{NH}_{3} \mathrm{PbBr}_{3}$ hybrid perovskite single}

\author{
crystals
}

\author{
Smaïl AMARI I', Jean-Marie VERILHAC', Eric GROS D'AILLON', Alain IBANEZ' and Julien \\ ZACCARO
}

Grenoble Alpes University, CEALITEN, F38000 Grenoble, France

2Grenoble Alpes University, CNRS, Grenoble INP, Institut Néel, BP166, 38042 Grenoble Cedex 09 (France)

Grenoble Alpes University, CEALETI, F38000 Grenoble, France

Organic-Inorganic Halide Perovskite, hybrid perovskite, $\mathrm{CH}_{3} \mathrm{NH}_{3} \mathrm{PbBr}_{3}, \mathrm{MAPbBr}_{3}, \mathrm{Crystal}$ Growth, Crystalline quality, structural defects

\begin{abstract}
Methylammonium lead tribromide $\left(\mathrm{CH}_{3} \mathrm{NH}_{3} \mathrm{PbBr}_{3}\right)$ single crystals has gained a growing attention in the past few years due to their use as model material to investigate relevant intrinsic perovskite properties, and for their potential applications for radiation detection. Their study has been facilitated by the ease and speed of fabrication of millimetric single crystals through a simple protocol of unseeded Inverse Temperature Crystallization (ITC). In this study, we show that such growing conditions suffer from both insufficient reproducibility regarding crystal quality and
\end{abstract}


low yield of single crystal obtention. In particular, we observed that more than the half of crystals obtained by this technique are polycrystals. The structural defects of the rest single crystals obtained have been characterized by cross polarized light, surface chemical etching to reveal dislocations, X-ray diffraction, ICP-MS, and H-NMR. The results reveal a strong variability of crystals regarding to internal strains and dislocation densities. Such defects can further severely impact the electronic transport properties of these materials. A more robust and reproducible protocol is proposed based on seeded growth combined with appropriate temperature profile selected from continuous crystal growth monitoring. A clear improvement in crystal quality is reached with higher transparency, minimized internal strains and a low dislocations density in the range of $10^{4}$ to $10^{5} \mathrm{~cm}^{-2}$.

\section{INTRODUCTION}

Thanks to their high absorption from the visible wavelengths up to high energy range, ${ }^{1,2}$ high charge carrier mobility - charge carrier lifetime product $(\mu . \tau){ }^{3.4}$ and ease of growth in solution, hybrid organic-inorganic perovskites (HOIP) are materials of interest for optoelectronic applications such as solar cells,,$^{5,6}$ light-emitting diodes, and visible or X-ray photodetectors..$^{8.9}$ Moreover, the perovskites single crystals of chemical formula $\mathrm{CH}_{3} \mathrm{NH}_{3} \mathrm{PbX}_{3}(\mathrm{X}=\mathrm{Br}, \mathrm{I})$ are helpful models to investigate the intrinsic optoelectronic properties of this class of materials. Saidaminov et $a l .^{10}$ established a growth procedure to obtain millimeter sized HOIP crystals in a few hours. The growth conditions take advantage of the retrograde solubility of these class of perovskites in polar aprotic solvents such as dimethylformamide (DMF) or gamma-butyrolactone $(\mathrm{GBL})$ at temperature below $100^{\circ} \mathrm{C}$. While such a fast protocol is capable of producing some crystals of high transparency, it has been shown more recently that decreasing the crystal growth kinetics would be more favorable to improve the optoelectronic properties." Indeed, single crystals with no visible defects can still contain significant amounts of structural defects such as dislocations which were found to be detrimental to charge carriers transport properties in the case of conventional inorganic semiconductors such as Si, Ge, GaAs, or CdTe..$^{12-16}$ By extension, these 
defects may be one potential root cause of the variability in mobility values reported in the literature for a given HOIP compound (Table S1). In this context, it is of paramount importance to define a robust and reproducible growth protocol leading to HOIP single crystals with low amount of structural defects.

Among the different perovskite compositions, $\mathrm{CH}_{3} \mathrm{NH}_{3} \mathrm{PbBr}_{3}\left(\mathrm{MAPbBr}_{3}\right)$ is an interesting candidate as the performances of the associated detection devices has demonstrated promising sensitivity for X-ray detection with competitive values compared with standard technologies. When grown under the Inverse Temperature Crystallization protocol (ITC) in DMF, the single crystals have a natural cubic shape that makes them convenient for the fabrication of devices. More important, $\mathrm{MAPbBr}_{3}$ exhibits no phase transition in the typical growth temperature range for HOIP $\left(20-100^{\circ} \mathrm{C}\right)$, which gives a wider leeway to find controllable and reproducible growing conditions. In this context, deeper control of crystal growth parameters and advanced characterizations of structural defects in $\mathrm{MAPbBr}_{3}$ single crystal is an important step toward better understanding of the intrinsic properties of this material.

For this purpose, the present study focuses on improving the growing protocol based on retrograde solubility in DMF, in order to increase the probability of fabrication of single crystals and improve their structural perfection. Only crystals free from any visible defects (so-called optical grade quality), were accurately analyzed to qualitatively and quantitatively characterize their structural defects by coupling several techniques: sample transparency was determined by transmission spectroscopy, strain fields were evidenced through polarized light microscopy and dislocation densities were estimated by etch-pits after a chemical etching. High Resolution XRay diffraction was used to quantify any crystallographic misorientations that could be generated by extensive strain fields or different growth sectors. Finally, impurity contents were determined by ICP-MS, 'H NMR, respectively for the inorganic and organic parts. Based on these advanced characterizations, it was possible to specify the structural defects present in single crystals grown 
under different conditions, and propose a more favorable growth procedure in terms of reproducibility and crystal quality.

\section{EXPERIMENTAL SECTION}

\section{Materials}

$\mathrm{CH}_{3} \mathrm{NH}_{3} \mathrm{Br}\left(>99\right.$ wt\%) was purchased from Greatcell Solar limited (Australia). $\mathrm{PbBr}_{2}$ (99.999 wt \%) and DMF (anhydrous, 99.8 wt \%) were purchased from Sigma-Aldrich. Salts were stored in a glove box under Ar atmosphere. Salts and solvents were used as received without any further purification.

\section{Solubility}

Millimeter-sized HOIP crystals have been elaborated by using the protocol described in ref 10 . For the solubility curve, we use $1 \mathrm{~mL}$ of fresh DMF as solvent. For each temperature, successive amounts of ground HOIP were added in the solution stirred at $500 \mathrm{rpm}$, and until excess is reached. We considered that excess was reached if the system was stable at least one hour after the last addition. This hypothesis was consolidated by using longer waiting time (overnight) for a couple of points. We defined the solubility limit for each temperature as the average between the last amount of perovskite before excess and first amount after excess with the error bars defined as the difference between the average and the concentration before and precursor saturation was observed.

\section{Solution Growths}

For the growth experiments, $\mathrm{MABr}$ and $\mathrm{PbBr}_{2}$ precursors were dissolved at 1:1 molar ratio in DMF in order to obtain $1 \mathrm{M}$ solutions of $\mathrm{MAPbBr}_{3}$ corresponding to an equilibrium temperature of $58^{\circ} \mathrm{C}$. All the growth solutions were first maintained at room temperature under stirring overnight, then filtered using PTFE filters with $0.2 \mu \mathrm{m}$ pore size to remove any insoluble particles. Three growth protocols were investigated labeled as unseeded step, seeded step and seeded linear, respectively. The one called unseeded step is derived from the ITC method of ref 10 where $1 \mathrm{M} \mathrm{MAPbBr}$ s solutions is abruptly brought from room temperature to $85^{\circ} \mathrm{C}$ (step temperature profile) leading to the spontaneous nucleation and growth of crystals. 
Experimentally, $1 \mathrm{~mL}$ solution was introduced in $5 \mathrm{~mL}$ glass flasks and then immersed into an oil bath at $85^{\circ} \mathrm{C}+/-0.5^{\circ} \mathrm{C}$. In this unseeded step protocol and with our experimental conditions, crystals spontaneously nucleated at the air-solution meniscus.

The two other tested protocols were seeded growths. Seeds were produced either by the unseeded ITC step protocol described previously or by slow evaporation of solutions at $60^{\circ} \mathrm{C}$. Then, the seeded growths were carried out in $5 \mathrm{~mL}$ of $\mathrm{MAPbBr}_{3}$ molar solutions $(1 \mathrm{M})$ introduced in $20 \mathrm{~mL}$ glass flasks. The oil bath temperature was controlled by a double jacket beaker at $+/-$ $0.01^{\circ} \mathrm{C}$. The flasks containing the solution were inserted in the oil bath keeping the solution meniscus slightly above the oil level in order to avoid any spurious nucleation. In these conditions, the temperature gradient across the glass flask keeps the temperature below spontaneous nucleation at the solution meniscus, or air-solution interface. On the other hand, as seed quality may dramatically influences the growth and structural defects of the produced crystals, seeds exhibiting sharp edges and regular flat faces were dispatched in two categories based on polarized light inspection: class A are high quality seeds (without significant strains) and class B are low quality seeds (with significant strains). Some examples of images of seeds of class A and B obtained between crossed polarizers are displayed in Figure S1. It should be noted that the seeds were manipulated with high precaution to minimize any additional strain that may come from the handling steps. The selected seeds were then mounted on silicon wafers with a silicone paste and placed at the bottom of the flask. The growth solution and the flask containing the seed were first separately thermalized for $1 \mathrm{~h}$ at $50^{\circ} \mathrm{C}$. Then the thermalized solution was quickly poured in the flask. At the initial stage, the seed is slightly dissolved, and then refaceted by setting the solution to achieve very reduced supersaturation (Figure S2). Then, for the growth from $65^{\circ} \mathrm{C}$ to $85^{\circ} \mathrm{C}$ two heating profile were applied: a step-like temperature increase (seeded step protocol) corresponding to a heating from $65^{\circ} \mathrm{C}$ to $85^{\circ} \mathrm{C}$ in 25 minutes, and a linear temperature profile (seeded linear protocol) involving a heating rate of $5^{\circ} \mathrm{C} / \mathrm{h}$. For all seeded protocols, the growth is constantly monitored by timed image acquisitions (with a typical 
resolution better than $100 \mu \mathrm{m})$ to estimate the growth rates and evolutions of the applied supersaturation from mass balance.

\section{Optical and polarized light microscopies}

Crystals were observed under moderate magnification $(\times 20)$. Samples were labeled single crystals if their habit presented no inward angles between adjacent facets. Furthermore, they were considered of optical grade quality if no crack, inclusion or heterogeneities were visible when observed with transmitted light under this magnification. The crystals were also observed in polarized light microscopy, in transmission mode and under moderate magnification $(\times 20)$ between crossed polarizers in order to determine the presence of strain fields in the bulk of crystals. Such strains could be generated either by the seed and the seed refaceting kinetic, or during the growth process (growth bands, inclusions, growth sectors boundaries).

\section{Transmission spectroscopy}

Transmission spectroscopy allowed determining the optical gap and linear attenuation coefficient (i.e. losses by residual absorption and diffusion per unit of length). The transmission spectra were recorded on a Perkins-Elmer Lambda 900 spectrometer, using an unpolarized beam. Polished samples of various thicknesses were mounted in front of an aperture with a diameter of $2 \mathrm{~mm}$. To better evidence the impact of the bulk of the samples, Fresnel losses and sample thickness were taken into consideration using the optical index $\mathrm{n}$ of the material from ref.17 leading to the linear attenuation coefficients calculated from the following equation: ${ }^{\text {: }}$

With:

$$
\alpha=\frac{1}{e} \cdot \ln \left(\frac{\frac{4 \cdot n(\lambda)}{(1+n(\lambda))^{2}}}{T(\lambda)}\right)
$$

- $\quad \alpha$ the linear attenuation coefficient

- $\quad$ e the thickness of the crystal

- $\quad T(\lambda)$ the measured transmittance at the wavelength $\lambda$

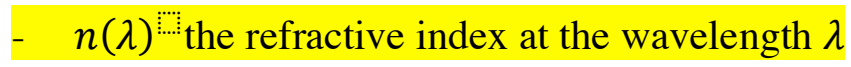




\section{Chemical Etching}

Chemical etching of natural growth faces reveals very localized strains (dislocations or grain boundaries) that facilitate dissolution. The etch pits patterns formed on the surface through a slight dissolution by DMF, made it possible to detect and count the dislocations by direct observation with an optical profilometer (Bruker GT Contour). Depending on their orientations and localizations, we could identify the source defects that create the dislocations in some cases.

\section{X-Ray Diffraction}

Structural defects are almost systematically associated with atomic plan distortions and/or interreticular spacing alteration. To estimate the crystal mosaicity, high resolution X-ray diffraction rocking curves measurements with a resolution of a few arc sec was used. To determine fine distortions to the long range order, X-ray diffraction rocking curves were

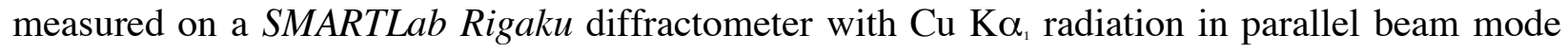
(curve multilayer mirror, four germanium monochromators) involving different slits on the detector (from $1 \mathrm{~mm}$ down to $50 \mu \mathrm{m}$ ) leading to an instrumental width of 12 arc sec. The incident beam covered the whole height of the crystal with an extension of around $400 \mu \mathrm{m}$ in the perpendicular direction. Rocking curves were measured at 2 different non overlapping positions. The Full Width at Half Maximum (FWHM) value indicated in the text is an average of the measurements. The full width at half maximum obtained for a diffraction peak combines the effect of atomic planes misorientation as well as distortions of interreticular spacing. For this purpose, coupled $\theta-2 \theta$ scans were also recorded in order to estimate the interreticular spacing variation alone.

\section{Inductively Coupled Plasma Mass Spectrometry (ICP-MS) and Nuclear Magnetic Resonance (NMR)}

ICP-MS and NMR were utilized to specify the main impurities and to determine their relative amounts in the as-grown crystals. For ICP-MS, an Agilent 7700x spectrometer was used in semiquantitative mode, involving $10 \mathrm{mg}$ of $\mathrm{MAPbBr}_{3}$ previously dissolved in $10 \mathrm{~mL}$ of a $5 \%$ (wt) 
aqueous solution of chlorhydric acid. 'H NMR spectra were recorded on a Bruker Avance 3 spectrometer working at $400 \mathrm{MHz}$ for proton, involving $20 \mathrm{mg}$ of $\mathrm{MAPbBr}_{3}$ samples previously dissolved in $600 \mu \mathrm{L}$ of DMSO-d6 using tetramethylsilane as internal reference.

\section{RESULTS AND DISCUSSION}

\section{Solubility}

The solubility curve displays in Figure 1 confirms the retrograde solubility of $\mathrm{MAPbBr}_{3}$ in $\mathrm{DMF}$ with a decrease solubility when the temperature increases. The solubility values obtained in this study are significantly higher than those determined in some previous work, ${ }^{19,20}$ and particularly at low temperatures $\left(20-60^{\circ} \mathrm{C}\right)$, where the solubility evolves sharply with temperature. We tentatively explain this discrepancy by a longer wait time before assuming equilibrium $(>1 \mathrm{~h})$ involved in our work compared to ref $19(30 \mathrm{~min})$ or ref 20 (duration not specified). It is important to note that the equilibrium temperature at $58{ }^{\circ} \mathrm{C}$ for $\mathrm{MAPbBr}_{3}$ molar solutions $(1 \mathrm{M})$, used as the starting point for all the following crystal growths, was always in perfect agreement with the solubility curve (Figure 1).

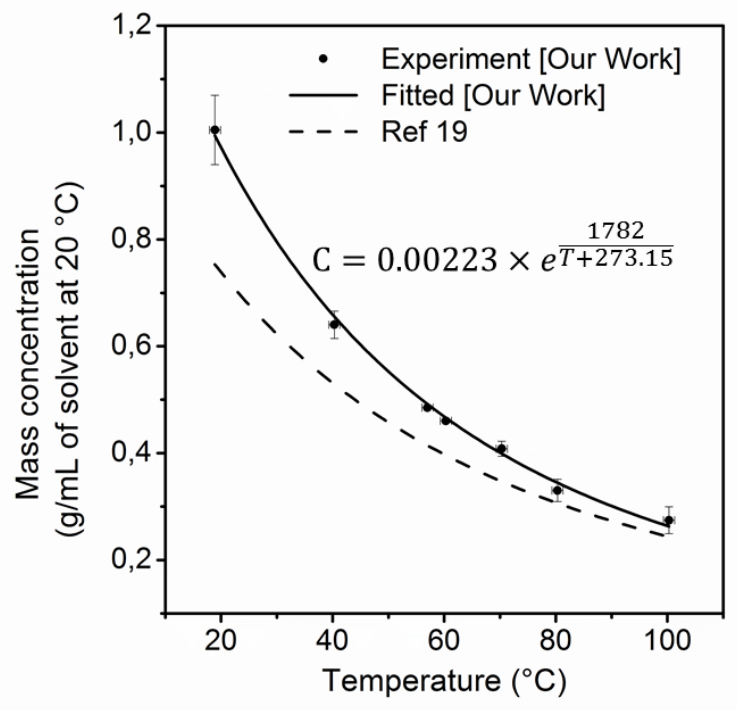

Figure 1. Solubility curve of $\mathrm{MAPbBr}_{3}$ in $\mathrm{DMF}$ 
The temperature dependence of the solubility limit of $\mathrm{MAPbBr}_{3}$ in $\mathrm{DMF}$ can be fitted with a Van't Hoff type equation: $\mathrm{C}=\mathrm{A} \times \exp (\mathrm{B} /(\mathrm{T}+273.15))$, with the term $\mathrm{A}$ linked to the entropy of dissolution and B the enthalpy of dissolution. This is consistent with the assumption that there is no change of predominant species in the liquid phase and in equilibrium with the solid phase between $20{ }^{\circ} \mathrm{C}$ and $100{ }^{\circ} \mathrm{C}$. Therefore, $\mathrm{MAPbBr}_{3}$ crystals can be advantageously grown without discontinuity over this full temperature range. In counterpart, this curve shows that, even at 100 ${ }^{\circ} \mathrm{C}$, the solubility and solubility slope remain high. Thus, even a slight change in temperature has a strong impact on the crystal growth. In such potentially unstable conditions, the temperature must be controlled with a high precision $\left(+/-0.01^{\circ} \mathrm{C}\right.$ or better) in order to carefully control the growth kinetics.

One alternative would be to find another solvent in order to decrease the solubility and solubility slope so as to make the process more tolerant to temperature variations. Nayak et al. ${ }^{21}$ showed that $\mathrm{MAPbBr}_{3}$ solubility can be decreased by adding formic acid in DMF. The solubility curves in DMF with different amounts of formic acid (Solubility curves: Figure S3) showed that both solubility and solubility slope could be significantly reduced. The curves can all be satisfactorily fitted using the same enthalpy of dissolution with concentration of formic acid in DMF up to 9.1\% vol. This is consistent with the fact that formic acid is not a solvent of $\mathrm{MAPbBr}_{3}$, and thus does not modify the enthalpy of dissolution. Indeed, formic acid alone cannot be used as solvent since $\mathrm{MAPbBr}_{3}$ powder in formic acid (98-100 \%) does not dissolve but decomposes in a few minutes into a white powder. In addition, for $9.1 \%$ vol. formic acid in DMF, a plateau in the solubility curve was observed below $60{ }^{\circ} \mathrm{C}$, indicating a change in predominant species in solution or in the stable solid-state phase at low temperature. This would significantly limit the temperature range for crystal growth in DMF containing large amounts of formic acid. Moreover, formic acid catalysis DMF degradation in presence of water; this degradation being even more pronounced when the temperature increases..$^{2}$ While formic acid allows lowering the solubility limit and solubility slope, it may introduce a significant source of unwanted variability into the growth process and should be avoided for growth runs lasting more than a few hours. 
For this reason, in this study all the crystal growths have been performed with pure DMF as solvent, in ambient atmosphere, and paying great attention to the temperature control.

\section{Unseeded step growths}

The unseeded step protocol has been investigated by growing more than 30 crystals following the procedure presented in ref 10 . The maximum dimensions of the crystals $\left(4.5 \times 4.5 \times 1.5 \mathrm{~mm}^{3}\right)$ were only limited by the volume of the solutions used $(1 \mathrm{~mL})$. On average, each growth run produced two crystals per flask. In our experimental conditions, all the crystals spontaneously nucleated at the air-solution meniscus before falling at the bottom of the flask. Among the 30 crystals, half were polycrystals. These polycrystals typically consisted of two or more individuals of about the same size originating from the nucleation site (Fig 2a). The other half representing single crystals were studied using polarized light microscopy and chemically etched to estimate their dislocation density. Polarized images showed that around half of them are heavily strained and only 3 crystals (about $10 \%$ of the total) present both low strains and have a significant size larger than $1.5 \times 1.5 \times 0.5 \mathrm{~mm}^{3}$ (Figure 2 ). For those 3 crystals, internal contrasts are clearly visible, which allows locating the nucleation site. Moreover, even for the best and largest achieved crystals, chemical etching showed that the top of the nucleation site is always associated with high dislocations density (Figure 3).

(a)

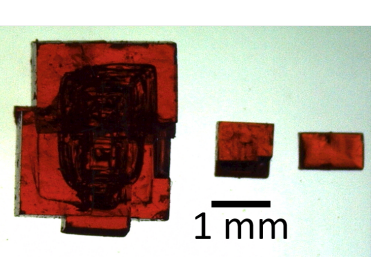

(b)

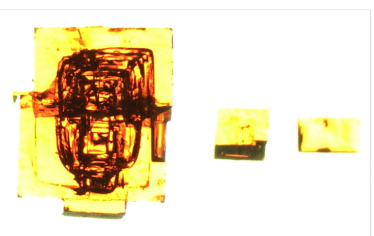

(c)

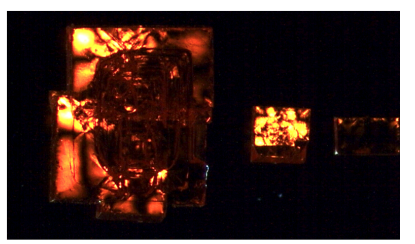

Figure 2. (a) Photograph of typical crystals obtained for the unseeded step protocol. (b) Same crystals observed with high intensity transmitted light and (c) between crossed polarizers. From left to right for each image: polycrystal, single crystal with strains, single crystal free of strain. 


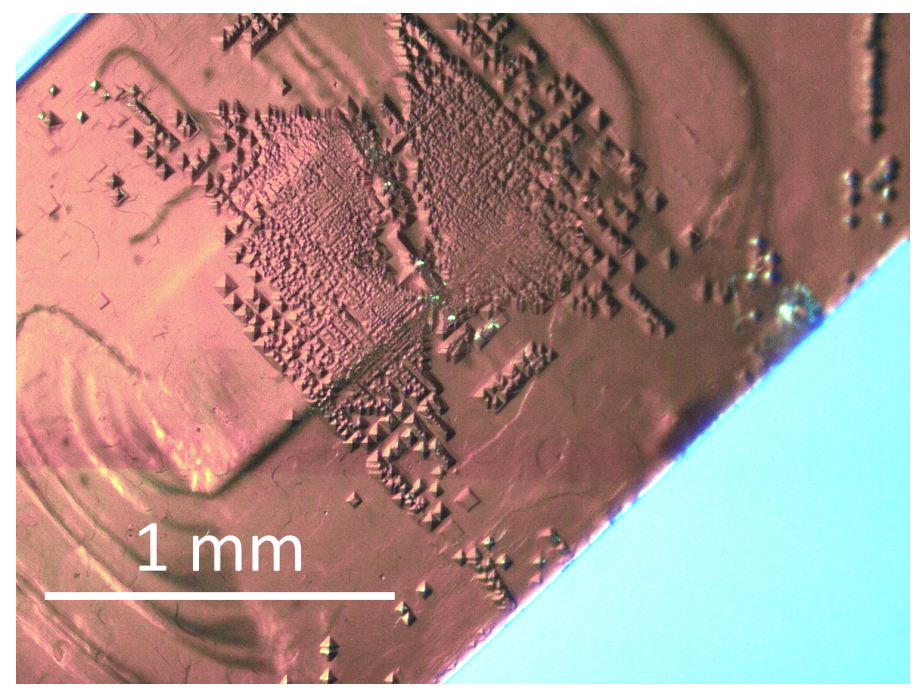

Figure 3. Optical image of etch pits on the top face of the best (strain free) and largest single crystals obtained with the unseeded step protocol. The high density of dislocations surrounding the nucleation site is clearly visible.

One of the main origins for the lack of reproducibility of this ITC protocol is the quality of the spontaneously nucleated seeds. Spontaneous nucleation leads to seeds of uncontrolled quality or even polycrystalline. The later is the main cause for obtaining polycrystals as is evidenced by the fact that the different individuals generally originate from the nucleation site. Even when a single crystal is grown, since strains and dislocations can propagate in the growing crystal, the seed strongly impact the final structural defects of the whole crystal volume.

Thus, while being relatively easy to achieve, unseeded protocol suffers from poor reproducibility in terms of crystal quality. In this sense, a careful control of the seeding step is mandatory in order to optimize the process reproducibility, increase the strain free volume of the crystals and reduce the dislocations density.

\section{Seeded step growths}

While unseeded protocols have been extensively used to grow $\mathrm{MAPbBr}_{3}$ (see for example ref $\mathrm{A}_{4,610,03-}$ $\left.{ }^{31}\right)$, the use of a seed is still poorly reported (see for example ref ${ }^{11,3233}$ ). The protocols presented 
hereafter detail the benefit of using a seed and a gentler temperature profile on the final structural defects of the crystal. At the beginning of the growing procedures, a mild dissolution of the seed is followed by its reconstruction under moderate supersaturation by a rise of the temperature above the equilibrium $\left(58^{\circ} \mathrm{C}\right) .25$ growth runs were performed with the seeded step protocol, and growth on a seed lead always to the formation of a crystal with typical dimensions above $4 \times 4 \times$ $2 \mathrm{~mm}^{3}$. The binocular observation revealed that only 9 crystals were polycrystals while the 16 others were single crystals, confirming that the seeded growth increases the process reproducibility in the production of single crystals. Nevertheless, contrary to our expectations, we observed that all the low strained seeds (class A) have led to the growth of polycrystals, while all of the strained seeds (class B) gave optical grade single crystals. Visible transmission spectroscopy performed on the crystals showed quite high transmittance only for the second kind of crystals (Figure S9). Thus, the derived linear attenuation coefficients (removing the contribution of Fresnel losses) in the transparency window (above $580 \mathrm{~nm}$ ) is found to be significantly lower for crystals grown from strained seeds than from unstrained seeds (Figure 4).

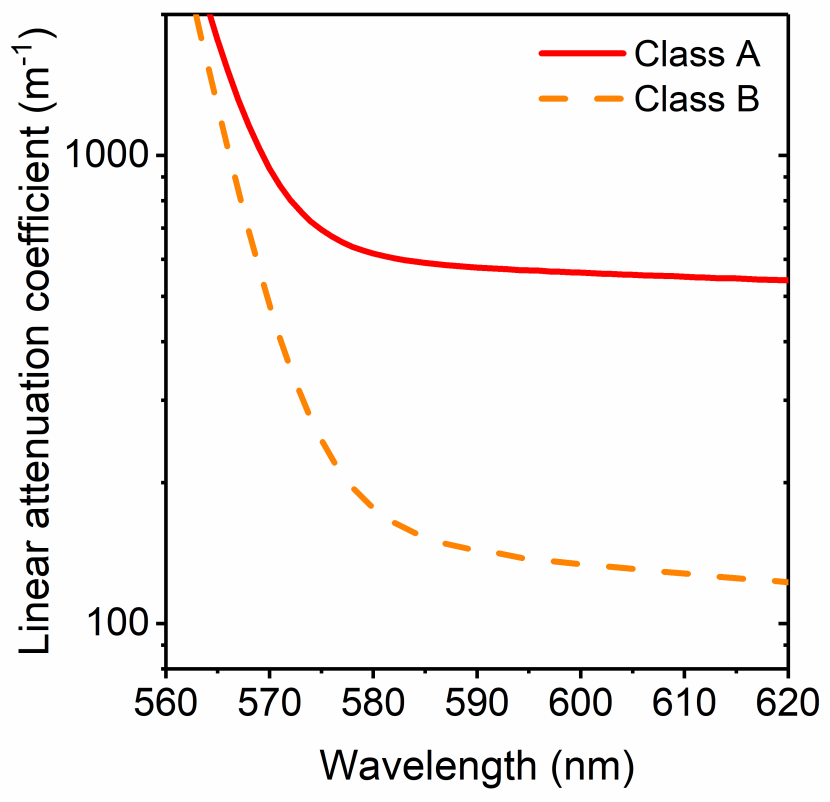


Figure 4. Linear attenuation coefficient for crystal grown from seeds of class A (weakly strained), and B (highly strained), and by the use of the seeded step protocol

This counter-intuitive result can be better understood by following the growth yields as a function of the temperature for crystals initiated from the two types of seeds. The growth yields presented in Figure 5 are estimated through the automatic crystal growth monitoring with timed image acquisitions by a camera (Figure S5). Figure 5 shows that strained seeds (class B) show higher crystallization yields compared to the unstrained seeds (class A). This difference is a typical feature of facetted growth. As the growth proceeds by the advancement of elementary steps on stable crystal faces, the growth yield is strongly dependent on the number of step sources. At low to moderate supersaturations, structural defects (mostly screw dislocation) act as step sources. Hence, low quality seeds have more step sources hence enhanced growth yield. Conversely, the high quality seeds show no noticeable growth before the temperature of $85^{\circ} \mathrm{C}$ is reached; there is negligible desaturation of the growth solution. So, high quality seeds grow under higher supersaturations ( $42 \%$ after $30 \mathrm{~min}$, Figure S6), favoring growth instabilities that generate structural defects detrimental to the optical transmission (scattering centers, incorporation of impurities).

Conducting growths at higher supersaturations is not incompatible with high crystal quality but it requires more stringent regulation of other parameters. For instance, at higher supersaturation morphological instabilities occur for lower inhomogeneity of the supersaturation over the crystal's faces. ${ }^{34}$ Also, if impurities are present higher supersaturations may favor their incorporation in the crystal lattice. In more extreme cases, if the impurity blocks the step advancement, high supersaturation can lead to pseudo dendritic growth, or "unorderly coverage" through secondary nucleation as observed for $\mathrm{NaCl}^{35}$ or more recently for $\mathrm{MAPbBr}_{3}{ }^{36} \mathrm{So}$, limiting the supersaturation and the supersaturation variation throughout the growth is critical to maintain optimal growth conditions and improve the crystal quality. This was confirmed in the case of the linear temperature profile presented below. 


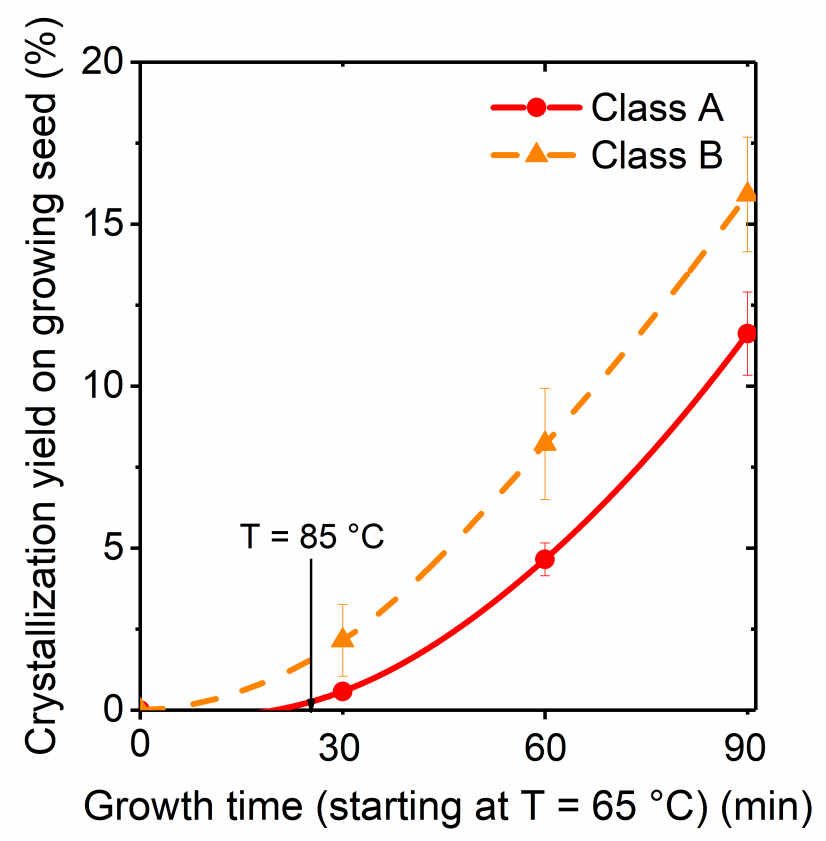

Figure 5. Crystallization yield on different seed qualities for crystals grown with a seeded step protocol ( 3 crystals from seed class A, and 3 crystals from seed class B).

These results demonstrate that in the case of the fast step-temperature profile used in the ITC protocol, the use of a strained seed tends to improve the reliability of the process. This process is however intrinsically limited since the optimal seeds to produce single crystals are of limited quality which ultimately reduces the achievable quality of the grown crystals.

\section{Seeded Linear Growths}

In order to take full advantage of seeded growth using seeds with minimal strains, we modified the temperature profile to lower the supersaturation. The temperature increase should be slow enough to allow unstrained seeds to grow significantly and thus desaturate the solution to avoid reaching too high supersaturations. The most straightforward way to achieve it, is by using a linear temperature profile with a suitable temperature increase rate. We found that a temperature increase rate of $+5^{\circ} \mathrm{C} / \mathrm{h}$ (linear profile) was well adapted. Indeed, no major difference in growth rate and crystallization yield was observed between strained and unstrained seeds with such 
linear profile (Figure 6a). This is a clear indication that under these conditions, the growth rates are no longer driven by the solution desaturation but more favorably by the supersaturation limited by the linear profile. In that case, the growth kinetics becomes seed quality independent and all crystals grown were single-crystals (Figure S4).

(a)

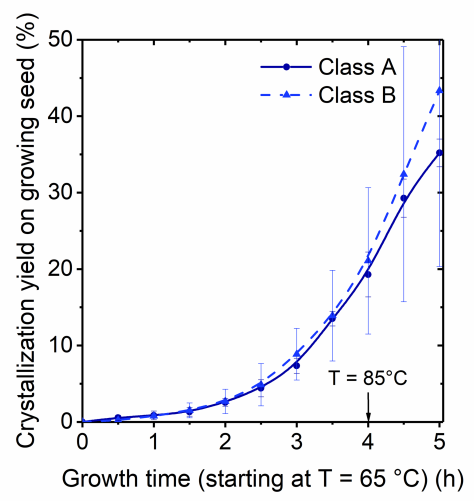

(b)

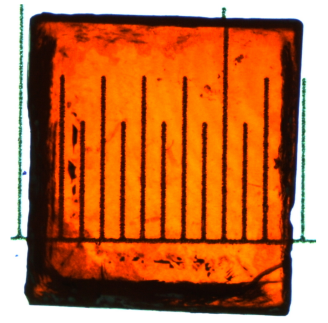

(d)

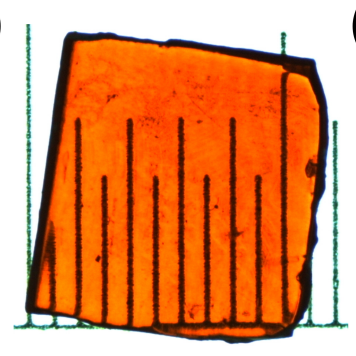

(c)

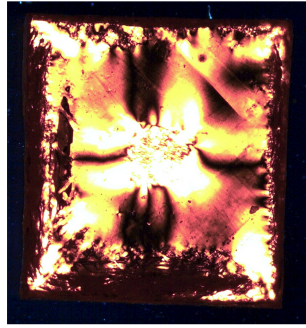

(e)

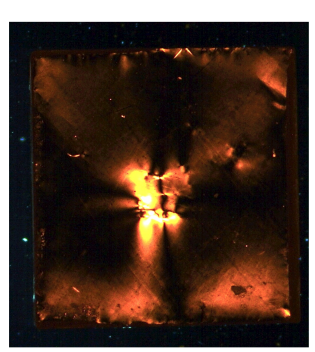

Figure 6. (a) Crystallization yield for growths with linear temperature profile on seed class A and class B. (b) Photograph and (c) cross polarized image of polished single-crystals grown from seeded linear protocol and seed of class B. (d) Photograph and (e) cross polarized image of polished single-crystals grown from seeded linear protocol and seed of class A.

Because the supersaturation is limited throughout the growth (Figure S6), no growth instability occurs, particularly in the early stages. Contrary to the step-temperature profile, unstrained seeds lead to optical grade single crystals instead of polycrystals (Figure 6d). Moreover, characterizations under crossed polarizers light clearly reveals the benefits of using the combination of linear temperature profile with weakly strained seeds. Crystals obtained from low strained seeds are significantly less constrained when compared to the ones grown from the highly strained seeds (Figure 6c,e, Figure 7). It has been shown that lattice strains in polycrystalline layer of $\mathrm{CH}_{3} \mathrm{NH}_{3} \mathrm{PbI}_{3}$ could enhance the ionic migration, ${ }^{37}$ and increase the defect 
concentrations that induce non-radiative recombination..$^{38}$ In this sense, reducing the internal strains in $\mathrm{MAPbBr}_{3}$ single crystal may be an important way to further improve their optoelectronic properties. Moreover, thanks to etch pit patterns (Figure 8), the benefit of the growth protocol optimization is also confirmed by the estimation of the dislocation density. The typical values extracted from 6 crystals are in the range of $10^{4}-10^{5} \mathrm{~cm}^{-2}$, with a $40 \%$ decrease in etch pit density on crystals initiated from seeds class A. The average results for all crystals are represented in Figure S7. These values lie in the same level as CdTe crystals commercially used for gamma-ray imaging. ${ }^{39}$ To our knowledge, it is the first time that dislocation density is estimated for HOIPs single crystals.

To further quantify the quality achieved with the linear temperature profile and a high quality seed, a single crystal grown under these conditions was characterized by High Resolution X-Ray diffraction rocking curves measurements. The X-Ray spot has a width of $50 \mu \mathrm{m}$ width and length higher than the lateral dimension of the crystal. It appears that the FWHM of (100) reflection measured in 2 non-overlapping positions of the sample lead to almost identical values of FWHM with an average of 28.3 arc sec (instrumental width 12 arc sec) (Figure 9a), which is significantly lower than the values previously reported in the literature. ${ }^{11.3233}$ Moreover, given the values of FWHM obtained in the couple $\theta-2 \theta$ scans (Figure $9 b$ ), the broadening of the rocking curves are mainly due to strains, and not to misorientation. However, it is to be noted that for such low energies X-Ray photons, the depth of $\mathrm{MAPbBr}_{3}$ contributing to the measured diffraction peaks is at best $50 \mu \mathrm{m}$. The rocking curve measurements were performed after chemical etching, providing relaxed surface free from any strain that may have been generated during handling. 
(a)

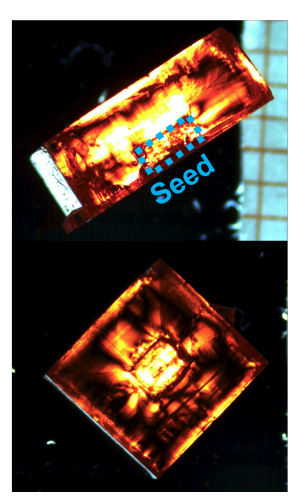

(b)

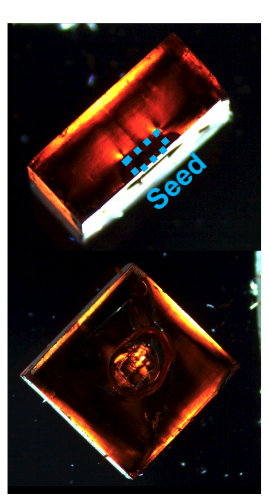

Figure 7. Crystals observed between crossed polarizers. (a) Best single crystal grown using the seeded step protocol (side and top views). (b) Best single crystals grown using the linear temperature profile (side and top views).

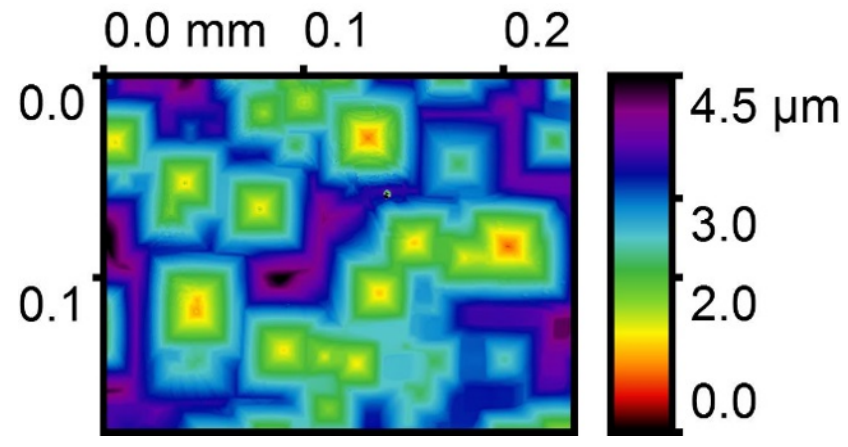

Figure 8. Typical etch pits pattern revealed by chemical etching of the surface of $\mathrm{MAPbBr}_{3}$ single-crystals and observed by optical profilometry. 
(a)

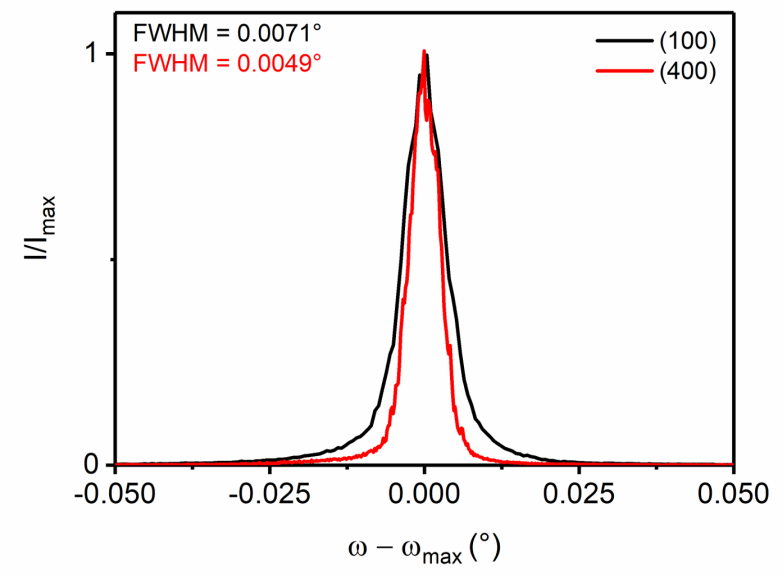

(b)

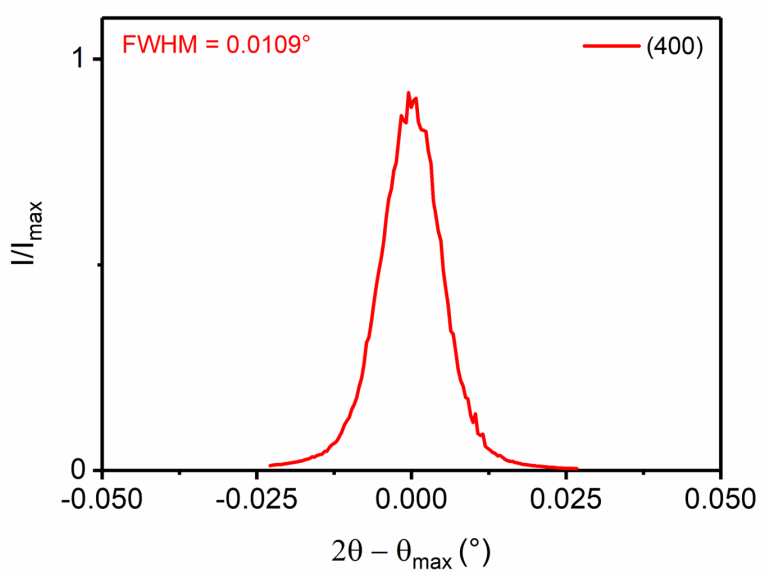

Figure 9. Rocking curves of a non-polished single crystal grown with the linear temperature profile. (a) High resolution X-Ray rocking curves for (100) and (400) reflections. (b) Coupled $\theta$ $2 \theta$ scans.

Finally, transmission spectroscopy showed a decrease of the linear attenuation coefficient for crystals grown with the linear temperature profile, independently of the grade of seed used (Figure S8).

All the above-mentioned characterizations give good evidence of extended structural defects, but they might not give a good indication regarding point defects such as impurities, which can have a very negative impact on charge carrier properties. ${ }^{36}$ To estimate and compare the impurity contents of grown crystals with the two temperature profiles, step and linear, we used ICP-MS 
and $\mathrm{H}$ NMR analyses. For both temperature profiles, the impurity content was below the detection threshold of ICP-MS for atomic number below 18 (1-5 ppm), and the content was below 1 ppm (in weight) for atomic number above 18 (Table S2). More precisely, no significant difference of quantity of any impurity was observed between the two profiles: a few dozens of ppb of Ag, Cs, and Tl, and a few hundreds of ppb of Bi. Regarding the 'H NMR, the only organic impurities detected are traces of DMF (Figure S10). They were observed for crystals obtained with the two temperature profiles tested, which could indicate the presence of scattering centers involving DMF.

Based on these results, we could argue that the difference of linear attenuation observed between the samples (from step and linear temperature profiles), cannot be attributed to residual absorption by some impurities. Measurements are under way to estimate if this difference can be attributed to scattering in the bulk and identify potential scattering centers. At this stage, there is no evidence of an eventual impact of the growth protocol or growth speed on the impurity content in the final single crystal. In this study, different growth speeds were used with values ranging from $3 \mathrm{mg} / \mathrm{h}$ to $60 \mathrm{mg} / \mathrm{h}$ for the first hour of growth. This is in agreement with the findings of ref 36. In that study, $\mathrm{MAPbBr}_{3}$ single crystals were grown at high growth rate with the unseeded step protocol using precursors of different purity. The resulting crystals were found to have no major difference in impurity content (TOF-SIMS). So it seems that while, high supersaturation is known to favor the incorporation of impurities in single crystals, $\mathrm{MAPbBr}_{3}$ even when grown at very high growth rate appears to be rather immune to impurity incorporation.

\section{CONCLUSION}

The impact of the $\mathrm{MAPbBr}_{3}$ crystal growth conditions on the process yield for single crystal obtention and on these crystals structural defects has been studied. The widely used unseeded inverse temperature crystallization protocol with DMF solvent is shown to be poorly reproducible and lead to uncontrolled crystal quality with a large proportion of polycrystals and highly strained single crystals. To overcome this issue, a seeded growth protocol has been proposed. The nature of temperature profile and the degree of seed constrains has be evaluated. 
The final crystal quality largely depends on the control of the crystallization kinetics, and especially on the level of supersaturation of the growth solution. The fast step temperature profile shows an intrinsic limitation due to the sharp increase in supersaturation when low constrained seeds were used, leading to uncontrolled growth that can even lead to polycrystals. We demonstrated that optimized parameters using a linear temperature profile $\left(5^{\circ} \mathrm{C} / \mathrm{h}\right)$ combined with a weakly strained seed increase significantly the reproducibility of the process and the quality of the as-grown crystals. As a result, single crystals are obtained at each run and the crystals show a higher transmittance, relaxed internal strains and a lower density of dislocations in the range of $10^{4}$ to $10^{5} \mathrm{~cm}^{2}$. Rocking curves measurements on these crystals reveals an unprecedented low diffraction width, below 30 arcsec.

Background on conventional semiconductors ( $\mathrm{Si}, \mathrm{CdTe} .$. ) showed that structural defects may have a huge impact on the optoelectronic performances. Even if such direct correlation is still missing in the case of $\mathrm{MAPbBr}_{3}$ and other HOIP single-crystals, we believe that the optimizations proposed in the present work will help to improve the performances of single crystal perovskite devices.

\section{ACKNOWLEDGEMENT}

The authors thank Sylvie MOTELLIER for ICP-MS analyses, Pierre-Alain BAYLE for his support on NMR analyses, and Stéphane GRENIER for high resolution X-Ray diffraction rocking curves measurement. The ICP-MS (Agilent 7700x) equipment is part of the NanoID platform supported by the French Investissement d'Avenir ANR-10-EQPX-39. The authors

thank the ATTRACT project from the European Horizon H2020 (Grant No. 777222) for financial support through the PerXI project.

\section{AUTHOR INFORMATION}

\section{Corresponding Author}

Give contact information for the author(s) to whom correspondence should be addressed 
Julien ZACCARO (julien.zaccaro@neel.cnrs.fr).

\section{Present Addresses}

\section{Author Contributions}

The manuscript was written through contributions of all authors. All authors have given approval to the final version of the manuscript.

\section{Funding Sources}

\section{Notes}

The authors declare no competing financial interest.

\section{ABBREVIATIONS}

DMF, N,N-dimethylformamide ; DMSO, dimethylsulfoxide ; FWHM, Full-Width Half

Maximum ; HOIP, Hybrid Organic-Inorganic Perovskite ; ITC, Inverse Temperature

Crystallization ; $\mathrm{MAPbBr}_{3}, \mathrm{CH}_{3} \mathrm{NH}_{3} \mathrm{PbBr}_{3}$; $\mathrm{NMR}$, Nuclear Magnetic Resonance ; ppb, part per billion ; PTFE, polytetrafluoroethane.

\section{REFERENCES}

(1) Babu, R.; Giribabu, L.; Singh, S. P. Recent Advances in Halide-Based Perovskite Crystals and Their Optoelectronic Applications. Cryst. Growth Des. 2018, 18 (4), 2645-2664.

(2) http://physics.nist.gov/cgi-bin/Xcom/Xcom2.

(3) Wei, W.; Zhang, Y.; Xu, Q.; Wei, H.; Fang, Y.; Wang, Q.; Deng, Y.; Li, T.; Gruverman, A.; Cao, L.; et al. Monolithic Integration of Hybrid Perovskite Single Crystals with Heterogenous Substrate for Highly Sensitive X-Ray Imaging. Nat. Photonics 2017, 11 (5), 315-321.

(4) Wei, H.; DeSantis, D.; Wei, W.; Deng, Y.; Guo, D.; Savenije, T. J.; Cao, L.; Huang, J. Dopant Compensation in Alloyed $\mathrm{CH}_{3} \mathrm{NH}_{3} \mathrm{PbBr}_{3-x} \mathrm{Cl}_{x}$ Perovskite Single Crystals for Gamma-Ray Spectroscopy. Nat. Mater. 2017, 16, 826-833.

(5) Liu, D.; Yang, J.; Kelly, T. L. Compact Layer Free Perovskite Solar Cells with 13.5\% Efficiency. J. Am. Chem. Soc. 2014, 136 (49), 17116-17122.

(6) Shi, D.; Adinolfi, V.; Comin, R.; Yuan, M.; Alarousu, E.; Buin, A.; Chen, Y.; Hoogland, S.; Rothenberger, A.; Katsiev, K.; et al. Low Trap-State Density and Long Carrier Diffusion in Organolead Trihalide Perovskite Single Crystals. Science 2015, 347 (6221), 519-522.

(7) Tan, Z.-K.; Moghaddam, R. S.; Lai, M. L.; Docampo, P.; Higler, R.; Deschler, F.; Price, M.; Sadhanala, A.; Pazos, L. M.; Credgington, D.; et al. Bright Light-Emitting Diodes Based on Organometal Halide Perovskite. Nat. Nanotechnol. 2014, 9 (9), 687-692. 
(8) Ahmadi, M.; Wu, T.; Hu, B. A Review on Organic-Inorganic Halide Perovskite Photodetectors: Device Engineering and Fundamental Physics. Adv. Mater. 2017, 1605242.

(9) Wei, H.; Huang, J. Halide Lead Perovskites for Ionizing Radiation Detection. Nat. Commun. 2019, 10 (1).

(10) Saidaminov, M. I.; Abdelhady, A. L.; Murali, B.; Alarousu, E.; Burlakov, V. M.; Peng, W.; Dursun, I.; Wang, L.; He, Y.; Maculan, G.; et al. High-Quality Bulk Hybrid Perovskite Single Crystals within Minutes by Inverse Temperature Crystallization. Nat. Commun. 2015, 6, 7586.

(11) Liu, Y.; Zhang, Y.; Zhao, K.; Yang, Z.; Feng, J.; Zhang, X.; Wang, K.; Meng, L.; Ye, H.; Liu, M.; et al. A $1300 \mathrm{~mm}^{2}$ Ultrahigh-Performance Digital Imaging Assembly Using HighQuality Perovskite Single Crystals. Adv. Mater. 2018, 30 (29), 1707314.

(12) Figielski, T. Dislocations as Electrically Active Centres in Semiconductors-Half a Century from the Discovery. J. Phys. Condens. Matter 2002, 14 (48), 12665.

(13) List, R. S. Electrical Effects of Dislocations and Other Crystallographic Defects in $\mathrm{Hg}_{0.78} \mathrm{Cd}_{0.22}$ Te n-on-p Photodiodes. J. Electron. Mater. 1993, 22 (8), 1017-1025.

(14) Mahajan, S. Defects in Semiconductors and Their Effects on Devices. Acta Mater. 2000, $48(1), 137$.

(15) Kurtz, A. D.; Kulin, S. A.; Averbach, B. L. Effect of Dislocations on the Minority Carrier Lifetime in Semiconductors. Phys. Rev. 1956, 101 (4), 1285.

(16) Mataré, H. F. Defect Electronics in Semiconductors; Wiley-Interscience: New York, 1971.

(17) He, C.; Zha, G.; Deng, C.; An, Y.; Mao, R.; Liu, Y.; Lu, Y.; Chen, Z. Refractive Index Dispersion of Organic-Inorganic Hybrid Halide Perovskite $\mathrm{CH}_{3} \mathrm{NH}_{3} \mathrm{PbX}_{3}(\mathrm{X} \mathrm{Cl}, \mathrm{Br}, \mathrm{I})$ Single Crystals. Cryst. Res. Technol. 2019, 54 (5), 1900011.

(18) Bohren, C. F.; Huffman, D. R. Absorption and Scattering of Light by Small Particles; Wiley-VCH: Weinheim, 2004.

(19) Saidaminov, M. I.; Abdelhady, A. L.; Maculan, G.; Bakr, O. M. Retrograde Solubility of Formamidinium and Methylammonium Lead Halide Perovskites Enabling Rapid Single Crystal Growth. Chem Commun 2015, 51 (100), 17658-17661.

(20) Ding, J.; Zhao, Y.; Du, S.; Sun, Y.; Cui, H.; Zhan, X.; Cheng, X.; Jing, L. Controlled Growth of $\mathrm{MAPbBr}_{3}$ Single Crystal: Understanding the Growth Morphologies of Vicinal Hillocks on (100) Facet to Form Perfect Cubes. J. Mater. Sci. 2017, 52 (13), 7907-7916.

(21) Nayak, P. K.; Moore, D. T.; Wenger, B.; Nayak, S.; Haghighirad, A. A.; Fineberg, A.; Noel, N. K.; Reid, O. G.; Rumbles, G.; Kukura, P.; et al. Mechanism for Rapid Growth of Organic-Inorganic Halide Perovskite Crystals. Nat. Commun. 2016, 7, 13303.

(22) Lide, D. R.; Baysinger, G.; Chemistry, S.; Berger, L. I.; Goldberg, R. N.; Kehiaian, H. V. CRC Handbook of Chemistry and Physics. pp. 5.27-5.34.

(23) Xu, Q.; Wei, H.; Wei, W.; Chuirazzi, W.; DeSantis, D.; Huang, J.; Cao, L. Detection of Charged Particles with a Methylammonium Lead Tribromide Perovskite Single Crystal. Nucl. Instrum. Methods Phys. Res. Sect. Accel. Spectrometers Detect. Assoc. Equip. 2017, 848, 106-108.

(24) Rao, H.-S.; Li, W.-G.; Chen, B.-X.; Kuang, D.-B.; Su, C.-Y. In Situ Growth of $120 \mathrm{~cm}^{2}$ $\mathrm{CH}_{3} \mathrm{NH}_{3} \mathrm{PbBr}_{3}$ Perovskite Crystal Film on FTO Glass for Narrowband-Photodetectors. Adv. Mater. 2017, 29 (16), 1602639.

(25) Wei, H.; Fang, Y.; Mulligan, P.; Chuirazzi, W.; Fang, H.-H.; Wang, C.; Ecker, B. R.; Gao, Y.; Loi, M. A.; Cao, L.; et al. Sensitive X-Ray Detectors Made of Methylammonium Lead Tribromide Perovskite Single Crystals. Nat. Photonics 2016, 10 (5), 333-339.

(26) Liu, X.; Zhang, H.; Zhang, B.; Dong, J.; Jie, W.; Xu, Y. Charge Transport Behavior in Solution-Grown Methylammonium Lead Tribromide Perovskite Single Crystal Using $\alpha$ Particles. J. Phys. Chem. C 2018, 122 (26), 14355-14361.

(27) Yakunin, S.; Dirin, D. N.; Shynkarenko, Y.; Morad, V.; Cherniukh, I.; Nazarenko, O.; Kreil, D.; Nauser, T.; Kovalenko, M. V. Detection of Gamma Photons Using SolutionGrown Single Crystals of Hybrid Lead Halide Perovskites. Nat. Photonics 2016, 10 (9), 585-589. 
(28) Sarritzu, V.; Sestu, N.; Marongiu, D.; Chang, X.; Wang, Q.; Masi, S.; Colella, S.; Rizzo, A.; Gocalinska, A.; Pelucchi, E.; et al. Direct or Indirect Bandgap in Hybrid Lead Halide Perovskites? Adv. Opt. Mater. 2018, 6 (10), 1701254.

(29) Wei, T.-C.; Wang, H.-P.; Li, T.-Y.; Lin, C.-H.; Hsieh, Y.-H.; Chu, Y.-H.; He, J.-H. Photostriction of $\mathrm{CH}_{3} \mathrm{NH}_{3} \mathrm{PbBr}_{3}$ Perovskite Crystals. Adv. Mater. 2017, 29 (35), 1701789.

(30) Ecker, B. R.; Wang, C.; Wei, H.; Yuan, Y.; Huang, J.; Gao, Y. Intrinsic Behavior of $\mathrm{CH}_{3} \mathrm{NH}_{3} \mathrm{PbBr}_{3}$ Single Crystals under Light Illumination. Adv. Mater. Interfaces 2018, 5 (23), 1801206.

(31) Ge, C.; Hu, M.; Wu, P.; Tan, Q.; Chen, Z.; Wang, Y.; Shi, J.; Feng, J. Ultralow Thermal Conductivity and Ultrahigh Thermal Expansion of Single-Crystal Organic-Inorganic Hybrid Perovskite $\mathrm{CH}_{3} \mathrm{NH}_{3} \mathrm{PbX}_{3}(\mathrm{X}=\mathrm{Cl}, \mathrm{Br}, \mathrm{I})$. J. Phys. Chem. C 2018, 122 (28), 1597315978.

(32) Zhang, L.; Liu, Y.; Ye, X.; Han, Q.; Ge, C.; Cui, S.; Guo, Q.; Zheng, X.; Zhai, Z.; Tao, X. Exploring Anisotropy on Oriented Wafers of $\mathrm{MAPbBr}_{3}$ Crystals Grown by Controlled Antisolvent Diffusion. Cryst. Growth Des. 2018, 18 (11), 6652-6660.

(33) Liu, Y.; Zhang, Y.; Yang, Z.; Feng, J.; Xu, Z.; Li, Q.; Hu, M.; Ye, H.; Zhang, X.; Liu, M.; et al. Low-Temperature-Gradient Crystallization for Multi-Inch High-Quality Perovskite Single Crystals for Record Performance Photodetectors. Mater. Today 2019, 22, 67-75.

(34) Staynova, M.; Nanev, Chr. On the Morphological Instability of the Growing Crystals (IV). Cryst. Res. Technol. 1989, 24(10), 951-958.

(35) Sarig, S.; Tartakovsky, F. Crystal Habit Modifiers II. The Effect of Supersaturation on Dendritic Growth. J. of Cryst. Growth 1975, 28, 300-305.

(36) Tisdale, J. T.; Smith, T.; Salasin, J. R.; Ahmadi, M.; Johnson, N.; Ievlev, A. V.; Koehler, M.; Rawn, C. J.; Lukosi, E.; Hu, B. Precursor Purity Effects on Solution-Based Growth of $\mathrm{MAPbBr}_{3}$ Single Crystals towards Efficient Radiation Sensing. CrystEngComm 2018, 20 (48), 7818-7825.

(37) Zhao, J.; Deng, Y.; Wei, H.; Zheng, X.; Yu, Z.; Shao, Y.; Shield, J. E.; Huang, J. Strained Hybrid Perovskite Thin Films and Their Impact on the Intrinsic Stability of Perovskite Solar Cells. Sci. Adv. 2017, 3 (11), eaao5616.

(38) Jones, T. W.; Osherov, A.; Alsari, M.; Sponseller, M.; Duck, B. C.; Jung, Y.-K.; Settens, C.; Niroui, F.; Brenes, R.; Stan, C. V.; et al. Lattice Strain Causes Non-Radiative Losses in Halide Perovskites. Energy Environ. Sci. 2019, 12 (2), 596-606.

(39) Burgess, L.; Kumar, F. J.; Mackenzie, J. Orientation Dependence of Etch Pit Density in (111) and (211) CdZnTe Everson Etch. J. Electron. Mater. 2015, 44 (10), 3277-3282. 